\title{
1.
}

\section{ON A THEOREM IN THE GEOMETRY OF POSITION.}

[From the Cambridge Mathematical Journal, vol. II. (1841), pp. 267-271.]

We propose to apply the following (new ?) theorem to the solution of two problems in Analytical Geometry.

Let the symbols

$$
|\alpha|, \quad\left|\begin{array}{cc}
\alpha, \beta \\
\alpha^{\prime}, \beta^{\prime}
\end{array}\right|, \quad\left|\begin{array}{l}
\alpha, \beta, \gamma \\
\alpha^{\prime}, \beta^{\prime}, \gamma^{\prime} \\
\alpha^{\prime \prime}, \beta^{\prime \prime}, \gamma^{\prime \prime}
\end{array}\right| \text {, \&c. }
$$

denote the quantities

$$
\alpha, \alpha \beta^{\prime}-\alpha^{\prime} \beta, \alpha \beta^{\prime} \gamma^{\prime \prime}-\alpha \beta^{\prime \prime} \gamma^{\prime}+\alpha^{\prime} \beta^{\prime \prime} \gamma-\alpha^{\prime} \beta \gamma^{\prime \prime}+\alpha^{\prime \prime} \beta \gamma^{\prime}-\alpha^{\prime \prime} \beta^{\prime} \gamma, \text { \&c. }
$$

(the law of whose formation is tolerably well known, but may be thus expressed,

$$
\begin{aligned}
& |\alpha|=\alpha, \quad\left|\begin{array}{l}
\alpha, \beta \\
\alpha^{\prime}, \beta^{\prime}
\end{array}\right|=\alpha\left|\beta^{\prime}\right|-\alpha^{\prime}|\beta|, \\
& \left|\begin{array}{c}
\alpha, \beta, \gamma \\
\alpha^{\prime}, \beta^{\prime}, \gamma^{\prime} \\
\alpha^{\prime \prime}, \beta^{\prime \prime}, \gamma^{\prime \prime}
\end{array}\right|=\alpha\left|\begin{array}{ll}
\beta^{\prime}, \gamma^{\prime} \\
\beta^{\prime \prime}, \gamma^{\prime \prime}
\end{array}\right|+\alpha^{\mid}\left|\begin{array}{l}
\beta^{\prime \prime}, \gamma^{\prime \prime} \\
\beta, \gamma
\end{array}\right|+\alpha^{\prime \prime}\left|\begin{array}{ll}
\beta, & \gamma \\
\beta^{\prime}, & \gamma^{\prime}
\end{array}\right| \text {, \&c. }
\end{aligned}
$$

the signs + being used when the number of terms in the side of the square is odd, and + and - alternately when it is even.)

Then the theorem in question is

$$
\begin{aligned}
& \rho \alpha+\sigma \beta+\tau \gamma . ., \rho \alpha^{\prime}+\sigma \beta^{\prime}+\tau \gamma^{\prime} . ., \rho \alpha^{\prime \prime}+\sigma \beta^{\prime \prime}+\tau \gamma^{\prime \prime} . .|\quad| \rho, \sigma, \tau . .|| \alpha^{\prime \prime}, \beta^{\prime \prime}, \gamma^{\prime \prime} . . \\
& \rho^{\prime} \alpha+\sigma^{\prime} \beta+\tau^{\prime} \gamma . ., \rho^{\prime} \alpha^{\prime}+\sigma^{\prime} \beta^{\prime}+\tau^{\prime} \gamma^{\prime} . ., \rho^{\prime} \alpha^{\prime \prime}+\sigma^{\prime} \beta^{\prime \prime}+\tau^{\prime} \gamma^{\prime \prime} . .=\rho^{\prime}, \sigma^{\prime}, \tau^{\prime} . . \quad \alpha^{\prime}, \beta^{\prime}, \gamma^{\prime} . \\
& \rho^{\prime \prime} \alpha+\sigma^{\prime \prime} \beta+\tau^{\prime \prime} \gamma . ., \rho^{\prime \prime} \alpha^{\prime}+\sigma^{\prime \prime} \beta^{\prime}+\tau^{\prime \prime} \gamma^{\prime} . ., \rho^{\prime \prime} \alpha^{\prime \prime}+\sigma^{\prime \prime} \beta^{\prime \prime}+\tau^{\prime \prime} \gamma^{\prime \prime} . .\left|\rho^{\prime \prime}, \sigma^{\prime \prime}, \tau^{\prime \prime} . .\right| \mid \alpha, \beta, \gamma . . \\
& \text { c. }
\end{aligned}
$$


(This theorem admits of a generalisation which we shall not have occasion to make use of, and which therefore we may notice at another opportunity.)

To find the relation that exists between the distances of five points in space.

We have, in general, whatever $x_{1}, y_{1}, z_{1}, w_{1}$, \&c. denote

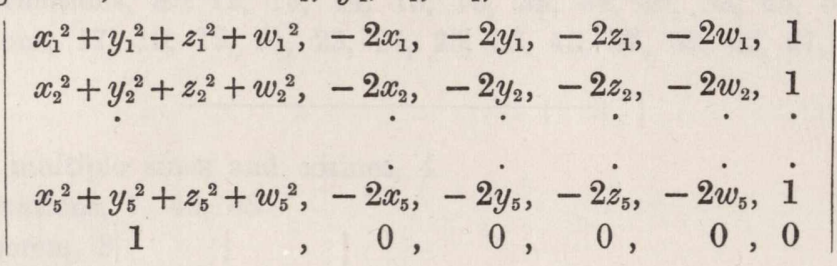

multiplied into

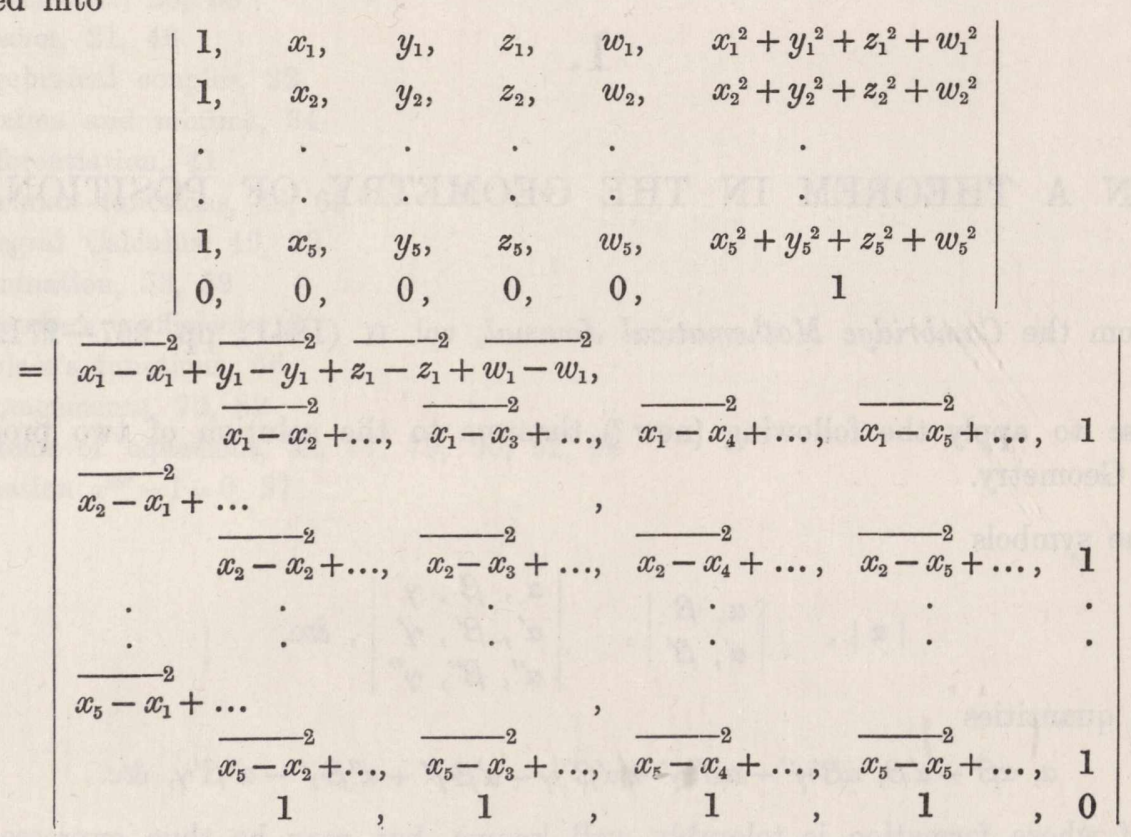

Putting the $w$ 's equal to 0 , each factor of the first side of the equation vanishes, and therefore in this case the second side of the equation becomes equal to zero. Hence $x_{1}, y_{1}, z_{1}, x_{2}, y_{2}, z_{2}$, \&c. being the coordinates of the points 1, 2, \&c. situated arbitrarily in space, and $\overline{12}^{2} \overrightarrow{13}^{2}$ \&c. denoting the squares of the distances between these points, we have immediately the required relation

$\begin{array}{cccccc}0, & \overline{12}^{2}, & \overline{13}^{2} & \overline{14}^{2} & \overline{15}^{2}, & 1 \\ \overline{21}^{2} & 0, & \overline{23}^{2} & \overline{24}^{2} & \overline{25,}^{2} & 1 \\ \overline{31}^{2} & \overline{32}^{2} & 0, & \overline{34,}^{2} & \overline{35,}^{2} & 1 \\ \overline{41}^{2} & \overline{42}^{2} & \overline{43,}^{2} & 0, & \overline{45,}^{2} & 1 \\ \overline{51}^{2} & \overline{52}^{2} & \overline{55,}^{2} & \overline{54,}^{2} & 0, & 1 \\ 1, & 1, & 1, & 1, & 1, & 0\end{array} \mid=$


which is easily expanded, though from the mere number of terms the process is somewhat long.

Precisely the same investigation is applicable to the case of four points in a plane, or three points in a straight line. Thus the former gives

$$
\left|\begin{array}{rrrrr}
0, & \overline{12}^{2}, & \overline{13}^{2}, & \overline{14}^{2} & 1 \\
\overline{21}^{2} & 0, & \overline{23}^{2} & \overline{24}^{2} & 1 \\
\overline{31}^{2} & \overline{32}^{2} & 0, & \overline{34}^{2} & 1 \\
\overline{41}^{2} & \overline{42}^{2}, & \overline{43}^{2} & 0, & 1 \\
1, & 1, & 1, & 1, & 0
\end{array}\right|=0 .
$$

The latter gives

$$
\left|\begin{array}{rrrr}
0, & \overline{12}^{2}, & \overline{13}^{2} & 1 \\
\overrightarrow{21}^{2} & 0, & \overline{23}^{2} & 1 \\
\overrightarrow{31}^{2} & \overline{32}^{2} & 0, & 1 \\
1, & 1, & 1, & 0
\end{array}\right|=0
$$

or expanding,

$$
\overline{12}^{4}+\overrightarrow{13}^{4}+\overline{23}^{4}-2 \cdot \overrightarrow{12}^{2} \overrightarrow{13}^{2}-2 \cdot \overrightarrow{13}^{2} \overrightarrow{23}^{2}-2 \cdot \overline{12}^{2} \overrightarrow{23}^{2}=0 ;
$$

which may be derived immediately from the equation

$$
\pm \overline{12} \pm \overline{13}= \pm \overline{23}
$$

and is the simplest form under which this equation, cleared of the ambiguous signs, can be put.

(The above result may be deduced so elegantly from the general theory of elimination, that notwithstanding its simplicity it is perhaps worth mentioning.)

Let

$$
x_{11}-x_{\mu}=\alpha, \quad x_{1 \prime}-x_{,}=\beta, \quad \overline{x_{1}-x_{\prime \prime}}=\gamma ;
$$

then

$$
\overline{12}^{2}=\gamma^{2}, \quad \overline{23}^{2}=\alpha^{2}, \quad \overline{31}^{2}=\beta^{2}, \quad \text { and } \alpha+\beta+\gamma=0 ;
$$

from which $\alpha, \beta, \gamma$ are to be eliminated. Multiplying the last equation by $\beta \gamma, \gamma \alpha$, $\alpha \beta$, and reducing by the three first,

$$
\begin{array}{r}
0 \cdot \alpha+\overline{12}^{2} \cdot \beta+\overline{31}^{2} \cdot \gamma+\quad \alpha \beta \gamma=0 \\
\overline{12}^{2} \cdot \alpha+0 \cdot \beta+\overline{23}^{2} \cdot \gamma+\quad \alpha \beta \gamma=0 \\
\overline{31}^{2} \cdot \alpha+\overline{23}^{2} \cdot \beta+0 \cdot \gamma+\quad \alpha \beta \gamma=0 \\
\alpha+\quad \beta+\quad \gamma+0 \cdot \alpha \beta \gamma=0
\end{array}
$$


from which, eliminating $\alpha, \beta, \gamma, \alpha \beta \gamma$ by the general theory of simple equations,

$$
\left|\begin{array}{rrrr}
0, & \overline{12}^{2} & \overline{13}^{2} & 1 \\
\overline{21}^{2} & 0, & \overline{23}^{2} & 1 \\
\overline{31}^{2} & \overline{32}^{2} & 0, & 1 \\
1, & 1, & 1, & 0
\end{array}\right|=0 .
$$

The (additional) equation that exists between the distances of five points on a sphere or four points in a circle, has such a remarkable analogy with the preceding, that they almost require to be noticed at the same time.

If $\alpha, \beta, \gamma, r$ be the coordinates of the centre, and the radius of the sphere, and $\delta=\alpha^{2}+\beta^{2}+\gamma^{2}-r^{2}$, we have immediately

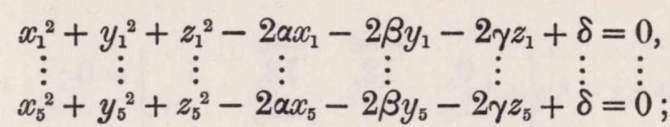

whence eliminating $\alpha, \beta, \gamma, \delta$,

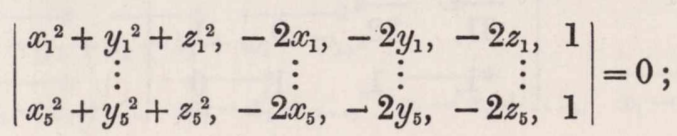

whence, multiplying by

we have immediately

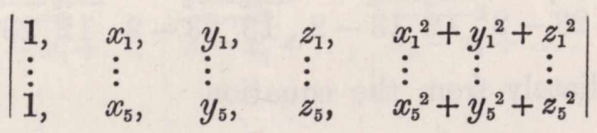

$$
\left|\begin{array}{ccccc}
0, & \overline{12}^{2}, & \overline{13}^{2} & \overline{14}^{2} & \overline{15}^{2} \\
\overline{21}^{2} & 0, & \overline{23}^{2} & \overline{24}^{2} & \overline{25}^{2} \\
\overline{31}^{2} & \overline{32}^{2} & 0, & \overline{34}^{2} & \overline{35}^{2} \\
\overline{41}^{2} & \overline{42}^{2} & \overline{43}^{2} & 0, & \overline{45}^{2} \\
\overline{51}^{2} & \overline{52}^{2} & \overline{53}^{2} & \overline{54}^{2} & 0
\end{array}\right|=0 .
$$

Forming the analogous equation for four points in a circle, and expanding, we readily deduce

$$
\overline{14}^{4} \overline{23}^{4}+\overline{12}^{4} \overline{34}^{4}+\overline{13}^{4} \overline{24}^{4}-2 . \overline{12}^{2} \overline{34}^{2} \overline{13}^{2} \overline{24}^{2}-2 . \overline{14}^{2} \overline{23}^{2} \overline{13}^{2} \overline{24}^{2}-2 . \overline{14}^{2} \overline{23}^{2} \overline{12}^{2} \overline{34}^{2}=0 \text {, }
$$

which is the rational, and therefore analytically the most simple form of

$$
\overline{12} \overline{34}+\overline{14} \overline{23}=\overline{13} \overline{24} \text {. }
$$

Euclid, B. vi., last proposition.

(It may be remarked that the two factors we have employed in the preceding eliminations, only differ by a numerical factor.) 